\title{
Labyrinthe
}

16 | 2003

Afrique(s)

\section{Le pétrole contre l'enclavement : un enjeu de la mondialisation au sud du Tchad}

\section{Géraud Magrin}

\section{(e) OpenEdition \\ Journals}

Édition électronique

URL : http://journals.openedition.org/labyrinthe/308

DOI : $10.4000 /$ labyrinthe.308

ISSN : 1950-6031

Éditeur

Hermann

Édition imprimée

Date de publication : 1 décembre 2003

Pagination : 19-34

Référence électronique

Géraud Magrin, «Le pétrole contre l'enclavement : un enjeu de la mondialisation au sud du Tchad », Labyrinthe [En ligne], 16 | 2003, mis en ligne le 06 juin 2008, consulté le 10 décembre 2020. URL

http://journals.openedition.org/labyrinthe/308 ; DOI : https://doi.org/10.4000/labyrinthe.308 


\title{
LE PÉTROLE CONTRE L'ENCLAVEMENT: UN ENJEU DE LA MONDIALISATION AU SUD DU TCHAD ${ }^{1}$
}

\author{
Géraud MAGRIN \\ Cirad-Tera/UMR Prodig
}

RÉSUMÉ: D'importants gisements de pétrole s'apprêtent à être exploités au sud du Tchad par un consortium réunissant des multinationales américaines et malaisiennes, sous l'égide de la Banque mondiale. Dans ce pays pauvre et enclavé de l'Afrique centrale, qui porte l'héritage d'une longue guerre civile, ces perspectives ont soulevé à la fois enthousiasme et inquiétude. L'or noir sera-t-il l'antidote tant attendu à l'isolement, à la division et au sous-développement? Ou bien, comme ailleurs en Afrique et dans le monde, ne ferat-il qu'alimenter la discorde, sur des bases ethniques ou religieuses? Ce texte, rédigé à la veille des travaux de construction de l'infrastructure d'exportation du pétrole tchadien, vise à en éclairer les enjeux géographiques et géopolitiques.

En Afrique comme ailleurs, les gisements pétroliers constituent un substratum géopolitique très inflammable. Malgré quelques exceptions, comme les oasis de prospérité et de paix du Gabon et de la Libye, nées de l'adéquation entre la faiblesse des populations et l'abondance des ressources, l'or noir a attisé bien des brasiers du continent. De l'Algérie au Nigeria, de l'Angola au Congo, en passant par le Cameroun, il a exercé des effets perturbateurs variés : désastres environnementaux (Nigeria), guerre civile à coloration ethnique ou religieuse pour la captation de la rente (Congo, Angola, Algérie), séparatisme (Nigeria), conflit frontalier (Nigeria/Cameroun). Jusqu'à une période récente, l'exploitation pétrolière en Afrique a privilégié les gisements littoraux. La création d'enclaves pétrolières off shore paraissait à la fois plus rentable et plus sûre. À la fin du $\mathrm{XX}^{\mathrm{e}}$ siècle, les perspectives haussières des marchés énergétiques à long terme incitent les grandes firmes transnationales des hydrocarbures à tenter l'aventure des bassins enclavés continentaux.

1. Ce texte utilise des informations collectées jusqu'en août 2000. 
Ainsi, la seconde moitié des années 1990 a vu la réactivation d'un vieux serpent de mer au Tchad. L'exploitation conjointe de deux gisements de pétrole, situés à Sédigui, au nord du lac Tchad, mais surtout à Doba, au cœur de la zone cotonnière méridionale (voir carte), pourrait permettre au pays de rompre avec son enclavement ainsi qu'avec le cercle vicieux de la pauvreté et du sous-développement. Le projet pétrolier alimentait alors un âpre débat entre partisans et adversaires de l'exploitation, à la fois au sud du Tchad, à l'échelle nationale, et, au-delà, par le canal de certaines ONG internationales, sur le théâtre de l'opinion occidentale. Si la question pétrolière a autant passionné, c'est qu'elle met en jeu une large gamme de problèmes brûlants. Autour des thèmes de l'environnement, de la gestion des ressources pétrolières, mais aussi des conditions internationales de son exploitation, ce sont les questions cruciales de l'enclavement du pays et de la place du Sud dans l'entité nationale tchadienne qui se trouvent posées.

Ces débats ne peuvent en effet s'interpréter sans référence aux dimensions géopolitiques sous-jacentes. La guerre civile qui a embrasé le Tchad au cours des années 1970 et 1980 a superposé des lignes de clivage internes sur des bases géographiques et religieuses (Nord contre Sud, musulmans contre chrétiens), puis très rapidement ethniques et personnelles (affrontements entre chefs de guerre du Bet ${ }^{2}$ ). Le brasier a été nourri de toutes parts par les pays voisins (Libye, Soudan, Nigeria) et par des puissances mondiales qui y défendaient des intérêts régionaux ou à plus large spectre (États-Unis, France, URSS et Chine). La question pétrolière met en jeu, pratiquement depuis ses origines, la même intrication d'échelles différentes. L'or noir semble ainsi enrichir la complexité géopolitique du Tchad en un mélange explosif.

\section{BRÈVE HISTOIRE D'UN VIEUX RÊVE PÉTROLIER}

\section{Le fardeau de l'enclavement}

Du début de la période coloniale à l'époque actuelle, les difficultés qui frappent l'importation des hydrocarbures pénalisent lourdement l'en-

2. Borkou Ennedi Tibesti : immense préfecture désertique du nord du pays. 


\section{Pétrole et conflits en Afrique}

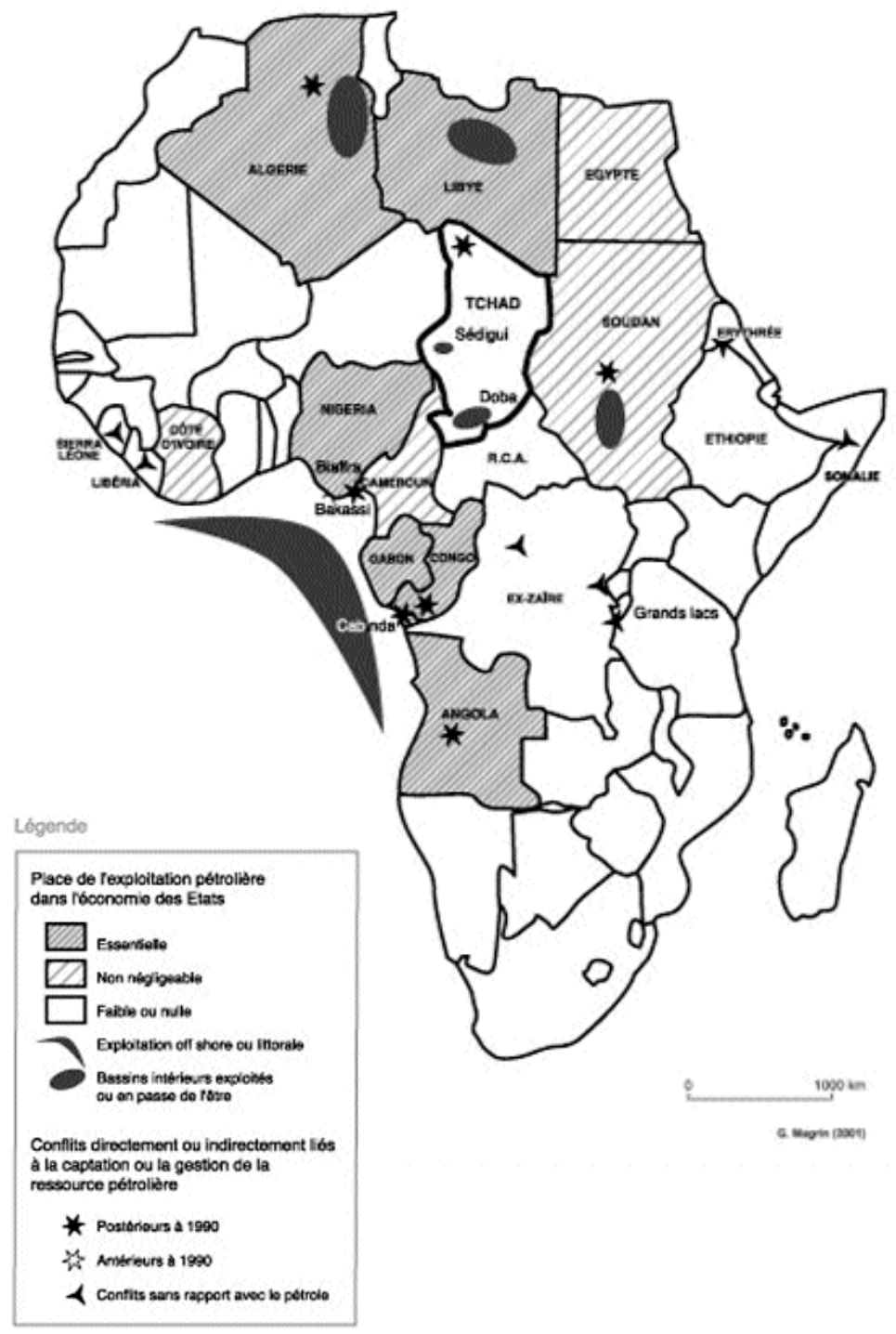


semble de la vie économique tchadienne. Elles affectent notamment la productivité, et donc la rentabilité de toutes les activités industrielles visant à valoriser sur place les matières premières issues de l'économie primaire.

Les premières recherches pétrolières en Afrique équatoriale française (AEF) remontent au début des années 1930, se focalisant sur les gisements littoraux. Dans le courant des années 1950, les recherches s'étendent à l'hinterland tchadien (Wagrenier, 1959: 6). Si les conclusions de ces premières études paraissent corroborées par les recherches ultérieures, alimentant la rumeur selon laquelle « les Français connaissaient l'existence du pétrole au Tchad avant l'indépendance », elles n'en présentent pas moins une certaine prudence. De même, l'intérêt économique de gisements situés au cour du continent, dans le contexte mondial d'abondance énergétique précédant les chocs pétroliers des années 1970, était sans doute peu évident aux yeux des responsables français. Devant l'indifférence française, le pouvoir tchadien se tourne vers la compagnie américaine Conoco, qui obtient un permis de prospection, et entreprend, à partir de 1969, une vaste campagne de recherches au-dessus du lac Tchad, puis de Doba au Salamat, en passant par Sarh. Un premier puits est creusé à Doba en septembre 1973.

\section{L'originalité technique et institutionnelle du projet pétrolier tchadien}

Après le retrait de Conoco, un consortium se constitue avec Shell, Chevron (1976), Exxon (1978) (Agir Ici, 1999: 11). En décembre 1992, après presque quinze ans d'immobilisme lié aux incertitudes politiques et militaires ${ }^{3}$, le consortium changeait à nouveau de composition. Le retrait de Chevron était compensé par la participation d'Elf (20\%), Shell et Exxon se partageant les parts principales (40\% chacun).

\footnotetext{
3. Une rébellion se développe à partir de 1967 dans certaines régions musulmanes du pays, contre le régime du président Tombalbaye, identifié aux Sara chrétiens du sud du pays. Elle s'organise autour du Front de libération national du Tchad (Frolinat). En 1975, un coup d'État mené par des militaires Sara renverse Tombalbaye. En 1979, le Frolinat balaie ce régime. Jusqu'en 1982, les différentes tendances du Frolinat s'entre-déchirent à N'Djamena. De 1982 à 1990, un de ses chefs, Hissène Habré, impose une dictature de fer, mate diverses rébellions, vainc la Libye de Kadhafi. En 1990, il est renversé par un de ses lieutenants, Idriss Déby. Tout en instaurant des apparences démocratiques, celui-ci doit depuis faire face à d'incessants mouvements de résistance armée sur différentes périphéries du territoire.
} 


\section{Le pétrole contre l'enclavement}

Depuis les années 1970, les perspectives d'exploitation pétrolière au Tchad concernent deux gisements de nature et d'importance différentes. Le premier, situé au Kanem, à Sédigui, à 350 kilomètres au nord-ouest de N'Djamena, contient des réserves limitées d'une huile légère, d'excellente qualité, qui pourrait pourvoir à l'essentiel des besoins tchadiens en hydrocarbures, mais ne pourrait justifier des exportations. Le second se trouve à proximité de Doba, au cœur de la zone soudanienne. Il recèle une huile lourde, dense, visqueuse, qui nécessite un raffinage approfondi avant utilisation, mais qui se prête à l'exportation, vue l'importance des réserves concernées. L'exploitation $\mathrm{du}$ premier vise donc essentiellement la satisfaction des besoins du marché intérieur, tandis que le second permettrait un renforcement des capacités financières de l'État.

Le projet d'exploitation du pétrole du bassin de Doba suppose un investissement total de l'ordre de 3,7 milliards de dollars, dont 1 milliard et demi pour le développement des champs pétroliers au Tchad et 2,2 milliards pour la construction d'un oléoduc de plus de 1000 kilomètres, qui doit exporter la production vers le littoral camerounais et, de là, vers les marchés mondiaux. Dès 1996, le principe d'un montage financier associant les membres du consortium, les deux États et la Banque mondiale est acquis. Les membres du consortium assureraient l'essentiel de l'investissement. La participation des États tchadiens et camerounais ne saurait se passer de l'engagement de la Banque mondiale. Celui-ci viserait à garantir le bon usage des ressources pétrolières, et ainsi à œuvrer sur le terrain dévolu à cette institution: la lutte contre la pauvreté. Du point de vue des pétroliers, la participation de la Banque mondiale revêt une importance financièrement symbolique, mais justement, et à ce titre, essentielle. Elle apporte l'indispensable caution pour la réalisation d'un investissement à haut risque.

Entre 1997 et 1999, devant les débats virulents engagés par la société civile tchadienne, et surtout des ONG internationales, la Banque exige davantage de garanties du gouvernement tchadien et du consortium en matière de respect des droits de l'homme et de l'environnement. En 1999, toutes les conditions semblent réunies. Le retour de la paix dans le sud du Tchad fin 1998 répond aux efforts réalisés par le consortium, attisant l'impatience des dirigeants tchadiens. C'est alors qu'intervient un coup de théâtre, avec l'annonce du retrait d'Elf 
et de Shell du consortium en novembre 1999, qui paraît compromettre encore une fois la réalisation du projet.

Dans un contexte marqué par la hausse du cours du pétrole sur les marchés mondiaux, la persévérance d'Exxon et du gouvernement tchadien est pourtant récompensée par une reconstitution rapide du consortium. Dans les premiers mois de l'an 2000, après quelques tergiversations, le nouveau consortium est constitué autour des américains Exxon (40\%) et Chevron $(25 \%)$ ainsi que du groupe malaisien Petronas $(35 \%)$. Peu après, le gouvernement tchadien signe avec la compagnie pétrolière soudanaise, Concorp International Limited, un accord pour l'exploitation des 15 millions de barils de Sédigui. Le 6 juin 2000, la Banque mondiale approuve enfin le projet, dont le financement sera assuré à 59,2\% par le consortium, à 12,9\% par le groupe de la Banque mondiale, le reste provenant de banques privées et du marché des capitaux.

\section{Le système d'exportation: des champs de pétrole à l'océan}

Les trois champs de Komé, Miandoum et Bolobo réunissent un milliard de barils de réserves prouvées, pour une production d'environ 240000 barils par jour, qui permettrait d'exporter entre 12 et 15 millions de tonnes annuelles. L'exportation du brut de Doba sera assurée par un oléoduc de 1061 kilomètres, dont 170 sur le territoire tchadien et 880 au Cameroun. Par ailleurs, alors que l'on prévoyait initialement pour l'oléoduc un diamètre de 24 pouces, on a finalement décidé en 1996 d'adopter un diamètre de 30 pouces ( 76 centimètres) [LAE, 1996], pouvant permettre d'évacuer une production supérieure aux 225000 barils quotidiens envisagés pour les seuls gisements de Doba. L'importance de l'investissement consenti par les groupes pétroliers laisse en effet de nombreux observateurs envisager la probabilité de nouvelles découvertes dans le prolongement du bassin de Doba. Celui-ci concerne des centaines de kilomètres, jusqu'au sud du Darfour soudanais en passant par la région de Sarh, le Salamat, voire le Nord-Est centrafricain.

Ainsi, la première infrastructure assurant en quelque sorte le désenclavement direct du Tchad ${ }^{4}$, c'est-à-dire sans rupture de charge, pour-

\footnotetext{
4. Après maints projets inaboutis, l'équipement de la « voie camerounaise » au début des années 1980 a permis un certain désenclavement du sud du bassin tchadien, grâce à une voie ferrée de 925 kilomètres, du port de Douala à Ngaoundéré, prolongée au nord par une route goudronnée de 745 kilomètres, qui atteint Kousséri, sur la rive camerounaise du Logone, face à N'Djamena.
} 


\section{Le pétrole contre l'enclavement}

rait être ce tube de 76 centimètres, cordon ombilical allongé sur près de 1100 kilomètres, des savanes méridionales du Tchad cotonnier à la grande forêt qui borde l'océan.

\section{LES PREMIERS EFFETS DE L'OR NOIR}

\section{En attendant la greffe pétrolière}

La grande pauvreté de la population et de l'État tchadien a constitué un sable propice aux mirages pétroliers. Acculés par les difficultés financières, fragilisés par des rébellions ou des guerres extérieures, les pouvoirs qui se succèdent dans la capitale tchadienne depuis l'indépendance ont entretenu l'espoir de jours meilleurs octroyés par l'avènement du dieu pétrole. Les Tchadiens ont espéré et désespéré, au rythme de l'intensité des crises politiques qui secouaient leur État.

Au début de l'année 2000, le cœur du pays pétrolier s'organise autour de la base de Komé, à une trentaine de kilomètres au sud-ouest de Doba. Elle est desservie par une piste d'aviation et par une route en latérite bien entretenue par le consortium. À partir de cette route viennent se greffer de nombreuses pistes secondaires, qui dessinent comme un labyrinthe, et conduisent tantôt à des puits, tantôt à des culs-de-sac exploratoires. Les puits apparaissent singulièrement modestes, comme de simples robinets fermés non encore reliés entre eux, entre lesquels les vaches paissent paisiblement.

La base matérialise cette intrusion d'un corps étranger dans les savanes soudaniennes du Tchad. Elle apparaît comme un grand carré de quelques hectares défriché dans la savane, entouré de hauts grillages, gardé à l'extérieur par un petit poste abritant une demi- douzaine de gendarmes tchadiens à l'apparence débonnaire. Elle a été installée en 1994. À l'intérieur de cette aire sont disposés quelques dizaines de conteneurs plus ou moins vides, des cuves à hydrocarbures, une trentaine de mobil-homes, qui servent à la fois de bureaux et de logements pour les deux ou trois dizaines de personnes qui vivent sur la base, dont trois ou quatre expatriés. La visite de l'enclave révèle l'étonnante extraterritorialité du lieu. À travers l'affirmation répétitive de codes et de normes propres à la grande firme transnationale transparaissent certaines obsessions qui détonnent dans le paysage tchadien, telles que la sécurité routière (limitation de vitesse, port de la ceinture de sécurité) 
ou encore la salubrité. À l'intérieur des mobil-homes, les murs sont couverts d'affiches évoquant le risque de paludisme: " Is today your malaria pill day? » L'alcool est interdit dans l'enceinte de la base, de même que les femmes qui ne travaillent pas pour le projet. En revanche, on trouve, en pleine brousse, un distributeur de crèmes glacées italiennes, un autre de thé et de café glacé. Une télévision diffuse les matchs de base-ball retransmis par $\mathrm{CNN}$, et des films américains à volonté.

À Moundou (120 000 habitants en 2000), principal centre urbain du Sud tchadien, ou encore à Doba (25 000 habitants), chef-lieu de préfecture pétrolière du Logone oriental, la manifestation la plus révélatrice des stratégies d'anticipation liées au pétrole résulte de la demande concernant le foncier. À Doba, en 1991, deux cents demandes de terrain étaient formulées, contre mille deux cents en 1998. Parmi les demandeurs, un grand nombre sont originaires du Sud vivant à N'Djamena, pour partie des fonctionnaires venant du Logone oriental. Ils se servent de leurs réseaux familiaux pour profiter des changements annoncés, et tirer une rente locative des constructions modestes que leurs revenus leur permettent de bâtir. Mais on trouve aussi des personnalités politiques et des commerçants d'envergure nationale, sans liens particuliers avec la région, qui souhaitent mettre en œuvre des stratégies spéculatives immobilières ou commerciales à une toute autre échelle. À côté d'un chef de canton du Kanem, on peut voir les noms de plusieurs ministres, celui du président de l'Assemblée nationale, le général Kamougué, dont le fief se situe pourtant beaucoup plus à l'est, dans le Moyen-Chari, celui encore d'Adoum Togoï, figure historique du Frolinat originaire du Bet, longtemps proche du président Déby.

Les zones rurales concernées directement par le projet se limitent à la zone d'exploitation des champs de pétrole et aux villages riverains de l'oléoduc. Les intenses tractations qui ont marqué la période 19952000 y ont eu des répercussions conséquentes. Dans un milieu rural marqué par une grande précarité socio-économique, la possibilité d'être « impacté » par le projet, c'est-à-dire indemnisé en compensation de l'occupation de terrains agricoles ou d'habitations, a été considérée comme une aubaine. 


\section{Le pétrole contre l'enclavement}

\section{Impacts économiques et géographiques : perspectives à court et moyen terme}

Sur le territoire tchadien, l'exploitation du pétrole affectera directement des espaces restreints. Les champs de production du pétrole s'étendent uniquement sur le territoire des trois cantons de Béro, Komé et Miandoum, qui comptait 28100 habitants en 1993. Cinq autres cantons, totalisant 63000 habitants en 1993, sont riverains du tracé de l'oléoduc (Esso, 1997 a.: 1-8). Ainsi, les huit cantons directement concernés par le système de production et d'exportation du pétrole de Doba rassemblaient 91100 habitants en 1993, soit seulement $3 \%$ de la population de la zone soudanienne à cette époque. Le déplacement et l'installation des personnes dont les habitations sont situées sur l'emplacement prévu pour réaliser l'infrastructure du projet ne concerneraient que cent cinquante familles environ, réparties dans soixante et un villages et cinq cantons.

L'oléoduc sera enterré à une profondeur moyenne d'un mètre (Esso, 1997 b. : 30). Au Tchad, mais surtout au Cameroun, le tracé a été choisi de manière à éviter les espaces les plus peuplés ou ceux présentant une fragilité écologique particulière. Une fois la construction terminée, les terrains seront rendus à leur utilisation antérieure, à quelques conditions près : il sera possible d'y pratiquer l'agriculture et l'élevage, mais la construction de bâtiments ou la plantation d'arbres y seront interdites, afin de permettre les opérations d'entretien et de réparation.

Durant les trois années et demie prévues pour la construction, le projet est d'employer en même temps 4000 personnes, dont $60 \%$ au Tchad - soit environ 2400 . Puis, au cours des vingt-cinq à trente années d'exploitation, les besoins en main-d'œuvre au Tchad sont estimés à 550 employés au maximum (Esso, 1997 a. : 1-4). Cela correspond au nombre d'emplois d'une des modestes usines régionales (brasserie du Logone de Moundou par exemple). Le consortium a souhaité que le gouvernement tchadien empêche le déplacement des populations vers le site et la création spontanée de villages, afin notamment d'éviter des heurts avec les autochtones (Esso, 1997 b. : 695). Dans le même but, un plan de recrutement cohérent a été préparé (Esso, 1997 a. : 1/21-23).

\footnotetext{
5. Plan de gestion de l'environnement, p. 69.
} 
La pauvreté du Tchad se lit dans l'indigence du budget de son État: 140 milliards de francs CFA, soit l'équivalent du budget d'une ville moyenne française comme Quimper, ou celui du film Titanic. Les contraintes à surmonter pour l'exploitation ont forcé le pouvoir tchadien à accepter des conditions médiocrement avantageuses, et à se contenter de percevoir 12,5\% du montant du brut (Sotinel, 1998). À partir de 2004, le budget tchadien se verrait augmenter de 40 à $75 \%$ par rapport à la période pré-pétrolière, ce qui constitue une augmentation à la fois conséquente et relativement modeste. Le discours selon lequel le pétrole viendrait résoudre toutes les difficultés du pays largement utilisé par des pouvoirs politiques impuissants à sortir le pays d'une économie de survie - risque d'être porteur de bien des désillusions. L'approbation du projet par la Banque mondiale a été précédée par l'annonce d'une stratégie gouvernementale pour l'utilisation des revenus du pétrole, qui doivent servir de façon prioritaire à la lutte contre la pauvreté. À côté des $10 \%$ épargnés pour les générations futures, $80 \%$ des recettes doivent être affectées à cinq secteurs prioritaires (Esso, 1997 b. : 71). Ceux-ci concernent la santé, l'éducation, le renforcement des infrastructures (routes, télécommunications), la promotion des activités productrices, notamment dans le secteur rural, et enfin la sécurité alimentaire.

Certains détracteurs de la filière cotonnière pronostiquent un rapide bouleversement de l'économie régionale, sous l'effet de l'or noir. La culture du coton, ingrate et peu rémunératrice, serait vouée à être délaissée par de nombreux paysans, attirés par les emplois plus valorisants suscités par la nouvelle orientation de l'économie. La vieille culture de rente laisserait place à une rente sans culture. Cette substitution sous-entend l'imminence d'une transformation radicale de l'économie, sur le modèle des États miniers d'Afrique centrale. Or, l'ampleur des débats soulevés par le projet pétrolier ne saurait dissimuler la relative modestie de ses retombées financières. Les revenus du pétrole de Doba transiteront par le budget de l'État tchadien, à partir de N'Djamena. On peut s'attendre à ce que la capitale concentre, comme toujours en pareil cas, une bonne partie de la rente pétrolière. La distribution de ces revenus à l'échelle nationale, saupoudrés en cinq secteurs prioritaires où les dépenses socio-éducatives non immédiatement productives occuperont une bonne place, viendra encore atténuer l'impact de l'exploitation pétrolière sur l'économie du Sud producteur. 


\section{Le pétrole contre l'enclavement}

Le pétrole ne révolutionnera probablement pas les structures de l'économie du Tchad, et encore moins celles de la zone soudanienne. En revanche, sa mise en exploitation pourrait servir de catalyseur à des évolutions récentes de l'économie du Sud tchadien. La construction de l'infrastructure se traduira d'emblée par une augmentation du volume d'activité économique et une accélération de la croissance des villes. Celles-ci s'accompagneront d'une amplification de la demande en produits agricoles et d'élevage.

Le pétrole ne devrait pas non plus remettre en cause l'organisation de la région méridionale. Les espaces riverains de l'oléoduc ne connaîtront qu'une perturbation ponctuelle, dans le temps et dans l'espace, mais également peu d'activité supplémentaire. Le reste de la zone soudanienne ne semble guère concerné directement. Seul Moundou paraît en mesure de valoriser à la fois sa relative proximité des gisements - une centaine de kilomètres de Komé - et ses fonctions de capitale régionale du Sud tchadien. L'ascétisme en vigueur sur les installations pétrolières devrait instituer Doba et Bébédjia en bases arrières, où les fonctions de loisir et d'hébergement (bars, auberges...) connaîtraient un épanouissement particulier. Mais leur petite taille, le faible niveau des équipements et des services qu'elles proposent orienteront plutôt vers Moundou les activités les plus valorisantes. La récupération du pétrole au service de l'opposition Nord/Sud ne peut donc correspondre qu'à une instrumentalisation politique très artificielle, tant les espaces directement impliqués sont restreints.

\section{UN PÉTROLE EXPLOSIF: ENJEUX GÉOPOLITIQUES ET DÉBATS IDÉOLOGIQUES}

\section{L'État et le consortium contre la société civile}

Les débats relatifs au projet pétrolier ont entretenu une atmosphère d'étrange effervescence entre 1997 et 2000 , où les procès d'intention nourris par les ONG et la société civile, frisant parfois l'hystérie, répondaient à la langue de bois humanisto-environnementaliste, nimbée de mauvaise foi, des multinationales pétrolières et du régime tchadien.

Dès la fin de l'année 1997, de nombreuses organisations écologistes mènent une farouche campagne d'hostilité au projet pétrolier tchadien. Elles ont convaincu la Banque mondiale de l'insuffisance de 
certaines études sur l'environnement, lesquelles prévoyaient, pour que le tracé de l'oléoduc évite le déplacement des populations, de lui faire traverser des zones écologiquement sensibles (Agir Ici, 1999: 46). L'efficacité du message contestataire a souvent résidé dans un parallèle effectué avec le funeste sort du pays Ogoni, dans le proche Nigeria, victime de l'alliance d'une multinationale pétrolière, d'ailleurs membre à l'époque du consortium tchadien, Shell, et d'une dictature militaire. La dévastation de l'environnement y répondait à une captation des revenus pétroliers du Sud du pays par une junte originaire du Nord, à l'image de l'avenir que les détracteurs du projet redoutent pour le Tchad (Sotinel, 1998). Si la pression des ONG internationales n'a pas suffi à décourager la Banque, elle a au moins instauré autour du projet un niveau de surveillance inédit pour ce type d'investissement, qui pourrait permettre d'en atténuer certains effets négatifs.

Les relations du consortium et du gouvernement tchadien avec la société civile nationale ont depuis le départ été caractérisées par une profonde méfiance. Longtemps, le dossier pétrolier a entretenu l'opacité qui entoure les affaires importantes de l'État tchadien. Loin du gouvernement, de la représentation nationale et de la presse, il restait l'affaire de quelques proches du chef de l'État. Les négociations avec le Cameroun au sujet du tracé du pipe-line et de la répartition de la rente se sont effectuées dans le plus grand secret. Les ONG de la zone soudanienne ont été contactées pour la première fois par le consortium en 1997, pour voir comment elles pourraient participer aux projets de développement accompagnant un programme qui avait été conçu sans elles (Djérambé, 1998). Par la suite, lors de deux séminaires nationaux tenus à Donia et à Bébédjia, des terrains d'entente ont été laborieusement trouvés, notamment autour des questions d'indemnisation des paysans expropriés.

L'approbation du projet par la Banque mondiale, le 6 juin 2000, clôt la contestation et inaugure l'ère pétrolière tant attendue au Tchad. Outre la diversité des acteurs impliqués, les mécanismes de contrôle des revenus pétroliers contribueront à l'originalité du projet d'exportation tchado-camerounais. Les déclarations rassurantes du consortium, des gouvernants tchadiens et de la Banque mondiale, pour lesquels toutes les mesures ont été prises afin que les ressources pétrolières soient gérées en toute transparence, pourront toujours répondre aux mises en garde alarmistes des adversaires du projet, qui dénoncent 


\section{Le pétrole contre l'enclavement}

à l'avance le détournement massif des richesses nationales au profit de quelques proches d'un régime clanique. Dans un État extrêmement pauvre comme le Tchad, la «politique du ventre » (Bayart, 1989) continuera probablement longtemps de conditionner la vie politique, d'autant que le régime du président Déby ne s'est guère distingué depuis ses origines par sa probité. Les revenus pétroliers ne seront pas gérés comme la banque de Genève au temps de Calvin, alors que la rente pétrolière induit des dysfonctionnements de comportement jusque dans les pays riches. Il n'empêche, si la mobilisation vigilante de la société civile ne se dément pas, les mesures mises en place pourraient contribuer à limiter les déviances et les détournements. Paradoxalement, on pourrait alors considérer que, au-delà de ses retombées financières, un des principaux mérites de l'or noir aura été de catalyser un mûrissement de la société civile, dans l'espace laissé vacant par une opposition politique interne démissionnaire.

\section{Les dimensions géopolitiques : pétrole et désenclavement}

Les stratégies des multinationales pétrolières intéressées par le projet d'exportation du brut tchadien ont été caractérisées, depuis les années 1960, par une certaine instabilité, partiellement calquée sur celle du pays. Au moins six entreprises ont participé aux activités d'exploration pétrolière ou de préparation de l'exploitation. Les changements de composition du consortium expriment les évolutions des stratégies mondiales de ces multinationales, qui confrontent leur estimation du risque tchadien avec l'état de leurs réserves et le niveau des cours mondiaux. Des considérations géopolitiques interviennent aussi pour rendre compte des recompositions successives du consortium.

Ainsi, une certaine lecture très polémique tend à considérer l'histoire récente de la politique française en Afrique comme étant tout entière régie par le « syndrome de Fachoda » (Agir Ici, 1999: 7), sous la forme d'une opposition systématique aux intérêts anglo-saxons sur le continent noir, et notamment dans les pays du « pré carré », c'està-dire les anciennes possessions coloniales françaises. Le terrain pétrolier constituerait un enjeu déterminant de cette opposition entre puissances. Au commencement de l'histoire pétrolière tchadienne, dans le courant des années 1960, l'intrusion de l'américain Conoco dans ce pays, consécutive au refus français d'étendre les prospections, aurait contribué à la brouille du président Tombalbaye avec l'ancienne métro- 
pole. Plus tard, le retournement de la France, qui abandonna Hissène Habré pour soutenir Idriss Déby, aurait procédé dans une large mesure du souhait d'imposer, avec Déby, une présence française au sein d'un consortium jugé trop anglophone (ibidem: 7). Lors du retrait de Chevron du consortium, en 1992, Esso et Shell auraient souhaité user de leur droit de préemption pour éviter la participation d'Elf (ibidem: 25). De fortes pressions françaises se seraient alors manifestées pour imposer l'entreprise nationale.

Le retrait d'Elf, en novembre 1999, fit l'effet d'une bombe à N'Djamena. Maladroitement annoncé au président Déby par l'ambassadeur de France en personne, il sembla confirmer l'étroitesse des liens entre cette société et la politique française en Afrique. Il fut perçu par les autorités tchadiennes comme un lâchage par l'ancienne puissance tutélaire. Mais ce retrait peut probablement s'interpréter autrement que ne le feraient les tenants du «syndrome de Fachoda ». Sa privatisation récente et son rachat par Total l'expliquent probablement, de même que le souci d'améliorer son image fortement détériorée par les épisodes de ses immixtions douteuses dans les affaires intérieures des États africains.

\section{Géopolitique du pétrole tchadien}

Au Tchad, les perspectives d'exploitation du pétrole de Doba s'inscrivent dans le contexte de l'opposition Nord/Sud, alimentant la théorie d'un complot du Nord visant à faire main basse sur les richesses du Sud. La combativité de la société civile face au projet pétrolier doit s'interpréter en considérant la nature de ses représentants. Constituée de membres des ONG, des Églises ou des médias, elle regroupe une majorité de jeunes cadres « sudistes », pour lesquels l'engagement dans la société civile constitue, plus ou moins consciemment, une forme de résistance à la situation politique du pays, qui leur concède une très faible place et les tient éloignés des structures de l'État. L'opposition fondamentale qu'ils vouent au système politique existant procède d'une grande méfiance vis-à-vis de ce qui vient du sommet de l'État. Dès lors, le projet pétrolier si ardemment défendu par la présidence ne pouvait qu'apparaître suspect.

Sur le terrain de la politique intérieure, l'opposant le plus irréductible au projet fut le député de Bébédjia, Yorongar, qui contribua par son activité, puis par son incarcération, à intéresser les ONG et certains médias internationaux au problème du pétrole tchadien. De fait, celui 


\section{Le pétrole contre l'enclavement}

qui aime à se faire appeler le « député des 300 puits » n'est pas opposé à toute exploitation du pétrole, car personne au Tchad ne peut véritablement être contre une ressource qui atténuerait la pauvreté du pays, mais il refuse la réalisation du projet dans les conditions politiques actuelles, qui ne peuvent d'après lui que favoriser la captation des revenus nouveaux par des dirigeants kleptocrates.

Enfin, au cours des années 1990, la région pétrolière a été un des berceaux des principales rébellions basées dans le sud du pays. Les actions du CSNDP de Moïse Ketté, en 1992-93, puis celle des FARF de Laokein Bardé, jusqu'en 1998, ont de ce fait toujours constitué un arrière-plan indissociable de la négociation sur le dossier pétrolier. La spécificité des rébellions méridionales au cours des années 1990 réside dans la disproportion entre leurs moyens militaires et leur efficacité médiatique: sans jamais avoir été en mesure de représenter un danger réel pour le pouvoir de N'Djamena, à la différence des troupes du MDD au lac Tchad jusqu'en 1996-97, ou de celles du MDJT ${ }^{6}$ dans le Tibesti depuis octobre 1998, elles parviennent à maintenir dans le Sud un climat d'insécurité qui a contribué à ralentir les négociations pétrolières.

Au terme de quelques années d'âpres débats et de préparation laborieuse, la participation de la Banque mondiale paraît sceller le destin pétrolier du Tchad. Ainsi, ces 1000 kilomètres d'oléoduc reliant les champs pétroliers de Doba au port de Kribi semblent consacrer le désenclavement du Sud tchadien, levant un des obstacles majeurs au développement du pays. Cette réalisation pose cependant plus de questions qu'elle n'en résout. L'établissement d'une enclave pétrolière au plus profond des savanes soudaniennes suffira-t-elle à désenclaver autre chose que les champs d'hydrocarbure qu'elle exploite?

Du point de vue du Tchad méridional, la mise en valeur du pétrole de Doba aura des effets ambigus. Plus encore que le coton, le pétrole procurera des revenus dépendants d'un capital étranger et de l'État basé à N'Djamena. La capitale captera l'essentiel d'une rente pétrolière qui profitera peu directement aux zones de production et au Sud

6. Mouvement pour la démocratie et la justice au Tchad. Le MDJT est le parti de Youssouf Togoïmi. 
en général. Si cette nouvelle rente est convenablement gérée dans une conjoncture d'ensemble favorable, elle contribuera néanmoins, au Sud, à amplifier des dynamiques antérieures, qui voient le vivrier marchand offrir une alternative à l'antique culture de rente du coton. L'huile précieuse pourrait ainsi lubrifier un système spatial à l'essence agricole plus diversifiée. Cela implique, néanmoins, que l'hypothèque géopolitique soit levée: que le pouvoir convainque le Sud, par ses actes, qu'il peut aussi profiter de l'or noir. Faute de quoi, le théâtre du conflit Nord/Sud pourrait s'embraser à nouveau, avec la Banque mondiale dans le rôle du pyromane involontaire.

\section{BIBLIOGRAPHIE}

Agir ici - Survie, Projet pétrolier Tchad-Cameroun. Dés pipés sur le pipe-line, Dossiers noirs de la politique africaine de la France $\mathrm{n}^{\circ} 13$, Paris, L'Harmattan, 1999, 63 p.

Bayart J.-F., L'État en Afrique. La politique du ventre, Paris, Fayart, 1989.

Djérambé A., «Le dossier pétrole et les ONG au Tchad », communication au séminaire d'information et d'échange sur le projet pétrolier et l'oléoduc Tchad-Cameroun, Yaoundé, 25 août 1998, 3 p.

Esso, a. Projet d'exportation tchadien, étude d'impact sur l'environnement. Partie tchadienne, Dames \& Moore, octobre 1997; b. Projet d'exportation tchadien. Plan de gestion de l'environnement, partie tchadienne, Dames \& Moore, novembre 1997, 172 p.

Lae (La Lettre Afrique Énergie) n 311, 4 décembre 1996.

Magrin G., Le Sud du Tchad en mutation. Des champs de coton aux sirènes de l'or noir, Paris, Sépia-Cirad, 2001, 427 p.

Sotinel T., «Le Tchad face aux sirènes pétrolières », in Le Monde, 3 septembre 1998, p. 13.

Tchad et Culture, « Dossier - le pétrole tchadien: controverse autour d'une exploitation », $\mathrm{n}^{\circ}$ 175, décembre 1998, p. 9-20.

Wagrenier Ph., Notes sur les possibilités de recherche pétrolières au Tchad, Institut équatorial de recherches et d'études géologiques et minières, Brazzaville, mars 1959, 11 p. 